\title{
Design, performance, and calibration of the CMS hadron-outer calorimeter
}

\author{
CMS HCAL Collaborations
}

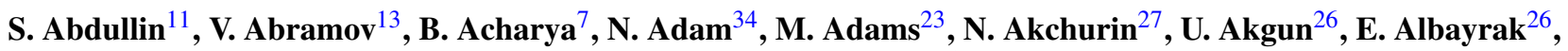

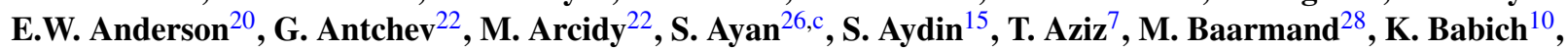
D. Baden $^{24}$, M.N. Bakirci ${ }^{15}$, Sudeshna Banerjee ${ }^{7}$, Sunanda Banerjee ${ }^{7, a}$, R. Bard ${ }^{23}$, V. Barnes ${ }^{38}$, H. Bawa ${ }^{6}$,

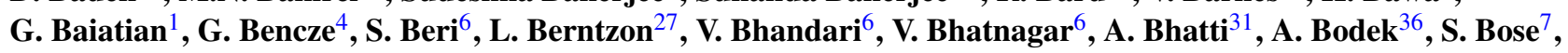
T. Bose ${ }^{35}$, H. Budd ${ }^{36}$, K. Burchesky ${ }^{23}$, T. Camporesi ${ }^{14}$, K. Cankoçak ${ }^{17,26}$, K. Carrell ${ }^{27}$, S. Cerci ${ }^{15}$, S. Chendvankar ${ }^{7}$, Y. Chung ${ }^{36}$, W. Clarida ${ }^{26}$, L. Cremaldi ${ }^{33}$, P. Cushman ${ }^{30}$, J. Damgov ${ }^{3,21}$, P. de Barbaro ${ }^{36}$, P. Debbins ${ }^{26}$, M. Deliomeroglu ${ }^{17}$, A. Demianov ${ }^{12}$, T. de Visser ${ }^{14}$, P.V. Deshpande ${ }^{7}$, J. Diaz $^{29}$, L. Dimitrov ${ }^{3}$, S. Dugad $^{7}$, I. Dumanoglu ${ }^{15}$, F. Duru ${ }^{26}$, I. Efthymiopoulos ${ }^{14}$, J. Elias ${ }^{21}$, D. Elvira ${ }^{21}$, I. Emeliantchik ${ }^{2}$, S. Eno ${ }^{24}$, A. Ershov ${ }^{12}$, S. Erturk ${ }^{15, \mathrm{~d}}$, S. Esen ${ }^{35}$, E. Eskut ${ }^{15}$, A. Fenyvesi ${ }^{5}$, W. Fisher ${ }^{34}$, J. Freeman ${ }^{21}$, S.N. Ganguli ${ }^{7}$, V. Gaultney ${ }^{29}$, H. Gamsizkan ${ }^{16}$, V. Gavrilov ${ }^{11}$, V. Genchev ${ }^{3}$, S. Gleyzer ${ }^{37}$, I. Golutvin ${ }^{10}$, P. Goncharov ${ }^{13}$, T. Grassi ${ }^{24}$, D. Green ${ }^{21}$, A. Gribushin ${ }^{12}$, B. Grinev ${ }^{19}$, M. Guchait ${ }^{7}$, A. Gurtu ${ }^{7}$, A. Murat Güler ${ }^{16}$, E. Gülmez ${ }^{17}$, K. Gümüşş ${ }^{27}$, T. Haelen ${ }^{36}$,

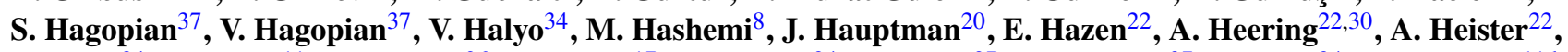

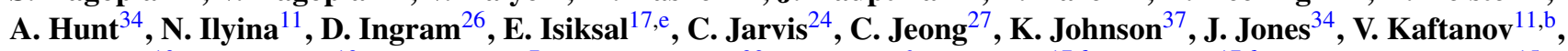
V. Kalagin ${ }^{10}$, A. Kalinin ${ }^{13}$, S. Kalmani ${ }^{7}$, D. Karmgard ${ }^{32}$, M. Kaur ${ }^{6}$, M. Kaya ${ }^{17, f}$, O. Kaya ${ }^{17, f}$, A. Kayis-Topaksu ${ }^{15}$, R. Kellogg ${ }^{24}$, A. Khmelnikov ${ }^{13}$, H. Kim ${ }^{27}$, I. Kisselevich ${ }^{11}$, O. Kodolova ${ }^{12}$, J. Kohli ${ }^{6}$, V. Kolossov ${ }^{11}$, A. Korablev ${ }^{13}$, Y. Korneev ${ }^{13}$, I. Kosarev $^{10}$, L. Kramer ${ }^{29}$, A. Krinitsyn ${ }^{13}$, M.R. Krishnaswamy ${ }^{7}$, A. Krokhotin ${ }^{11}$, V. Kryshkin ${ }^{13}$, S. Kuleshov ${ }^{11}$, A. Kumar ${ }^{6}$, S. Kunori ${ }^{24}$, A. Laasanen ${ }^{38}$, V. Ladygin ${ }^{10}$, E. Laird ${ }^{34}$, G. Landsberg ${ }^{34}$, A. Laszlo ${ }^{4}$,

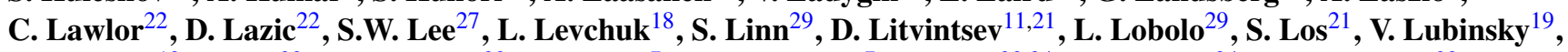

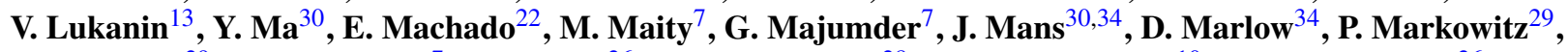
${\text { G. } \text { Martinez }^{29} \text {, K. Mazumdar }}^{7}$, J.P. Merlo ${ }^{26}$, H. Mermerkaya ${ }^{28}$, G. Mescheryakov $^{10}$, A. Mestvirishvili ${ }^{26}$, M. Miller ${ }^{26}$, A. Moeller ${ }^{26}$, M. Mohammadi-Najafabadi ${ }^{8}$, P. Moissenz $^{10}$, N. Mondal ${ }^{7}$, V. Mossolov $^{2}$, P. Nagaraj $^{7}$, V.S. Narasimham ${ }^{7}$, E. Norbeck ${ }^{26}$, J. Olson ${ }^{26}$, Y. Onel ${ }^{26}$, G. Onengut $^{15}$, C. Ozkan $^{16}$, H. Ozkurt $^{15}$, S. Ozkorucuklu ${ }^{17, g}$, F. Ozok ${ }^{26}$, S. Paktinat ${ }^{8}$, A. Pal ${ }^{4}$, M. Patil ${ }^{7}$, A. Penzo ${ }^{9}$, S. Petrushanko ${ }^{12}$, A. Petrosyan ${ }^{10}$, V. Pikalov ${ }^{13}$, S. Piperov ${ }^{3,21}$, V. Podrasky ${ }^{25}$, A. Polatoz ${ }^{15}$, A. Pompos ${ }^{38}$, S. Popescu ${ }^{27}$, C. Posch ${ }^{22}$, A. Pozdnyakov ${ }^{11}$, W. Qian ${ }^{23}$, R.M. Ralich ${ }^{28}$,

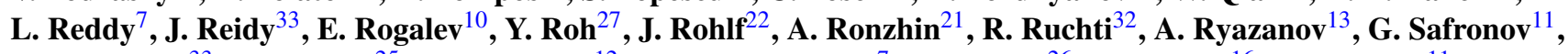
D.A. Sanders ${ }^{33}$, C. Sanzeni ${ }^{25}$, L. Sarycheva ${ }^{12}$, B. Satyanarayana ${ }^{7}$, I. Schmidt ${ }^{26}$, S. Sekmen ${ }^{16}$, S. Semenov ${ }^{11}$,

V. Senchishin ${ }^{19}$, S. Sergeyev ${ }^{21}$, M. Serin ${ }^{16}$, R. Sever ${ }^{16}$, B. Singh ${ }^{6}$, J.B. Singh ${ }^{6}$, A. Sirunyan ${ }^{1}$, A. Skuja ${ }^{24}$, S. Sharma ${ }^{7}$, B. Sherwood ${ }^{30}$, N. Shumeiko ${ }^{2}$, V. Smirnov ${ }^{10}$, K. Sogut ${ }^{15, h}$, N. Sonmez ${ }^{17, i}$, P. Sorokin ${ }^{18}$, M. Spezziga ${ }^{27}$,

R. Stefanovich ${ }^{2}$, V. Stolin ${ }^{11}$, K. Sudhakar ${ }^{7}$, L. Sulak ${ }^{22}$, I. Suzuki ${ }^{21}$, V. Talov ${ }^{13}$, K. Teplov ${ }^{12}$, R. Thomas ${ }^{27}$,

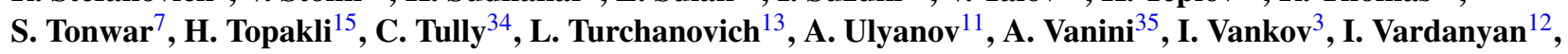

F. Varela ${ }^{22}$, M. Vergili ${ }^{15}$, P. Verma ${ }^{7}$, G. Vesztergombi ${ }^{4}$, R. Vidal $^{21}$, A. Vishnevskiy ${ }^{10}$, E. Vlassov ${ }^{11,14}$,

I. Vodopiyanov ${ }^{28}$, I. Volobouev ${ }^{27}$, A. Volkov ${ }^{13}$, A. Volodko ${ }^{10}$, L. Wang ${ }^{24}$, J. Werner ${ }^{34}$, M. Wetstein ${ }^{24}$, D. Winn ${ }^{25}$,

R. Wigmans ${ }^{27}$, J. Whitmore ${ }^{21}$, S.X. Wu ${ }^{22}$, E. Yazgan $^{27}$, T. Yetkin ${ }^{26}$, P. Zalan ${ }^{4}$, A. Zarubin ${ }^{10}$, M. Zeyrek ${ }^{16}$

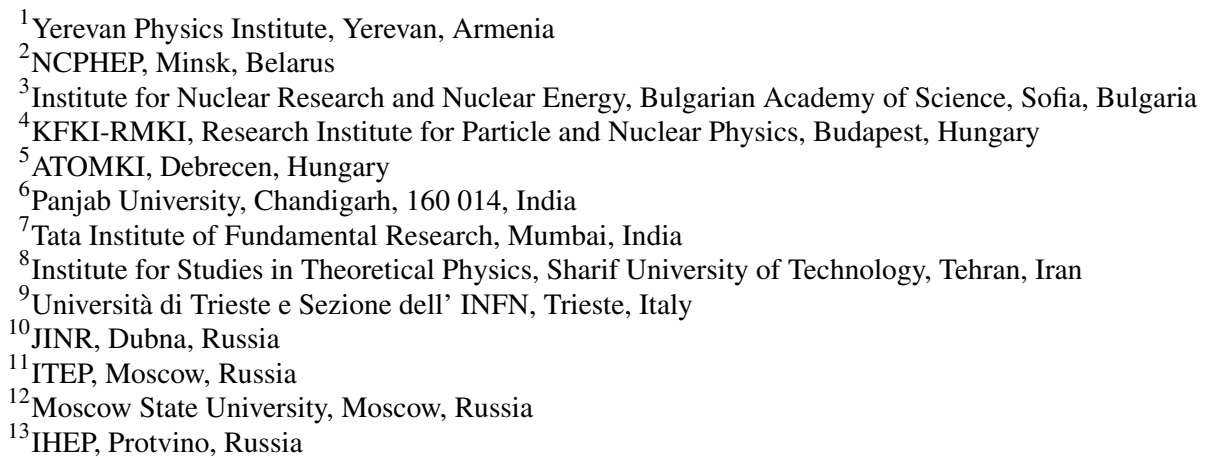


${ }^{14}$ CERN, Geneva, Switzerland

${ }^{15}$ Cukurova University, Adana, Turkey

${ }^{16}$ Middle East Technical University, Ankara, Turkey

${ }^{17}$ Bogazici University, Istanbul, Turkey

${ }^{18}$ KIPT, Kharkov, Ukraine

${ }^{19}$ Single Crystal Institute, Kharkov, Ukraine

${ }^{20}$ Iowa State University, Ames, IA, USA

${ }^{21}$ Fermi National Accelerator Laboratory, Batavia, IL, USA

${ }^{22}$ Boston University, Boston, MA, USA

${ }^{23}$ University of Illinois at Chicago, Chicago, IL, USA

${ }^{24}$ University of Maryland, College Park, MD, USA

${ }^{25}$ Fairfield University, Fairfield, CT, USA

${ }^{26}$ University of Iowa, Iowa City, IA, USA

${ }^{27}$ Texas Tech University, Lubbock, TX, USA

${ }^{28}$ Florida Institute of Technology, Melbourne, FL, USA

${ }^{29}$ Florida International University, Miami, FL, USA

${ }^{30}$ University of Minnesota, Minneapolis, MN, USA

${ }^{31}$ Rockefeller University, New York, NY, USA

${ }^{32}$ University of Notre Dame, Notre Dame, IN, USA

${ }^{33}$ University of Mississippi, Oxford, MS, USA

${ }^{34}$ Princeton University, Princeton, NJ, USA

${ }^{35}$ Brown University, Providence, RI, USA

${ }^{36}$ University of Rochester, Rochester, NY, USA

${ }^{37}$ Florida State University, Tallahassee, FL, USA

${ }^{38}$ Purdue University, West Lafayette, IN, USA

Received: 13 August 2008 / Published online: 10 October 2008 (C) Springer-Verlag / Società Italiana di Fisica 2008

\begin{abstract}
The Outer Hadron Calorimeter (HCAL HO) of the CMS detector is designed to measure the energy that is not contained by the barrel (HCAL HB) and electromagnetic (ECAL EB) calorimeters. Due to space limitation the barrel calorimeters do not contain completely the hadronic shower and an outer calorimeter (HO) was designed, constructed and inserted in the muon system of CMS to measure the energy leakage. Testing and calibration of the $\mathrm{HO}$ was carried out in a $300 \mathrm{GeV} / \mathrm{c}$ test beam that improved the linearity and resolution. $\mathrm{HO}$ will provide a net improvement in missing $E_{T}$ measurements at LHC energies. Information from $\mathrm{HO}$ will also be used for the muon trigger in CMS.
\end{abstract}

\footnotetext{
a e-mail: Sunanda.Banerjee@cern.ch

${ }^{\mathrm{b}}$ Deceased.

${ }^{\mathrm{c}}$ Now at University of Pennsylvania, Philadelphia, PA, USA.

${ }^{\mathrm{d}}$ At Niğde University, Niğde, Turkey.

e At Marmara University, Istanbul, Turkey.

${ }^{\mathrm{f}}$ At Kafkas University, Kars, Turkey.

${ }^{g}$ At Süleyman Demirel University, Isparta, Turkey.

${ }^{\mathrm{h}}$ At Mersin University, Mersin, Turkey.

${ }^{\mathrm{i} A t}$ Izmir Yüksek Teknoloji Enstitüsü, Izmir, Turkey.
}

\section{Introduction}

The Compact Muon Solenoid (CMS) experiment [1] at the Large Hadron Collider (LHC) at CERN is a multi-purpose detector optimised to look for signatures of the Higgs boson, super-symmetric particles and other new physics processes in $p p$ collisions at a centre of mass energy of $14 \mathrm{TeV}$. Signature for production of many of these new particles is large missing transverse energy, $E_{T}$. The momenta of all charged particles are measured using a high resolution tracker in a $4 \mathrm{~T}$ solenoidal magnetic field, while energies of all particles, charged as well as photons and neutral hadrons, are measured using electromagnetic and hadron calorimeters. Key elements in improving the missing transverse energy measurement are hermiticity of the calorimeter, excellent energy resolution and small energy leakage.

Figure 1 shows the longitudinal quarter view of the CMS detector. The hadron calorimeter [2], located behind the tracker and the electromagnetic calorimeter as seen from the interaction point, is a sampling calorimeter made of copper alloy ${ }^{1}$ absorber and plastic scintillators. The barrel hadron calorimeter (HB) has a polygonal structure. It is made out of two halves each $4.33 \mathrm{~m}$ long. A half barrel is made of 18 wedges each subtending $20^{\circ}$ in azimuth $\phi$. The HB has 16 scintillator layers designed to be hermetic with

\footnotetext{
${ }^{1}$ Cartridge brass \# 260 with $70 \%$ Copper and 30\% Zinc.
} 


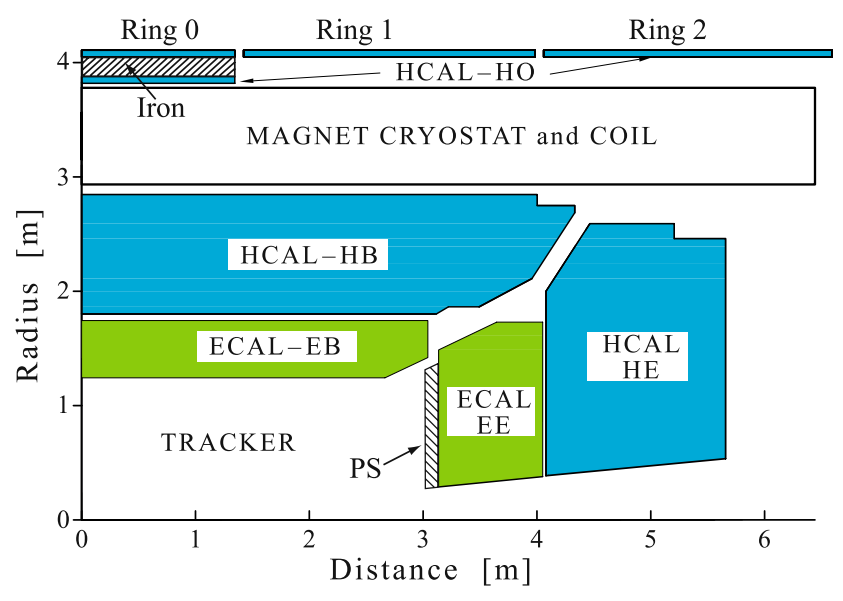

Fig. 1 Location of the hadron calorimeter in and around the CMS magnet. HCAL HB and HCAL HE are the hadron calorimeters. ECAL $\mathrm{EB}$ and ECAL EE are the electromagnetic calorimeters and PS is pre-shower detector

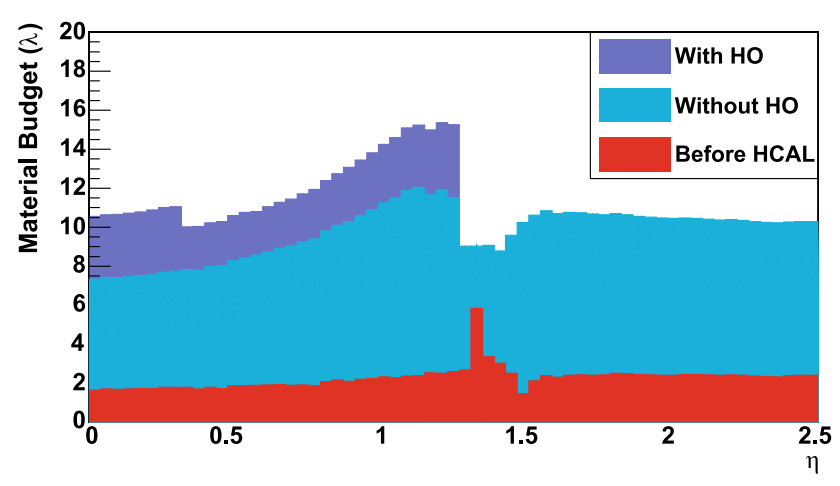

Fig. 2 Number of interaction lengths of the CMS calorimeter as a function of $\eta$. The two shaded regions correspond to the setups with or without the outer hadron calorimeter $(\mathrm{HO})$

minimal uninstrumented gaps. HB extends up to $|\eta| \approx 1.4$ ( $\eta=-\ln \tan \frac{\theta}{2}$, where $\theta$ is the polar angle).

$\mathrm{HB}$ is radially restricted between the outer extent of the electromagnetic calorimeter $(R=1.77 \mathrm{~m})$ and the magnet cryostat and coil $(R=2.95 \mathrm{~m})$. This constrains the total amount of material for the calorimeters EB plus HB to about 7.8 interaction lengths $\lambda_{\mathrm{I}}$ at $\eta=0$ as can be seen in Fig. 2 .

Extensive Monte Carlo simulations and test beam studies were carried out [3] to define the parameters of the hadron calorimeter. Figure 3 shows the fraction of events that are missing more than one third of the energy for a test beam of $300 \mathrm{GeV} / \mathrm{c}$ for $\mathrm{HB}$ alone. Adding the tracker and $\mathrm{EB}$, at $300 \mathrm{GeV} / \mathrm{c}$ nearly $3 \%$ of pion events gives rise to missing energy corresponding to $100 \mathrm{GeV}$. Small number of events with large leakage of energy lead to large fluctuation on an event by event basis and cannot be corrected offline. This unmeasured energy is the primary justification for the outer hadron $(\mathrm{HO})$ calorimeter.

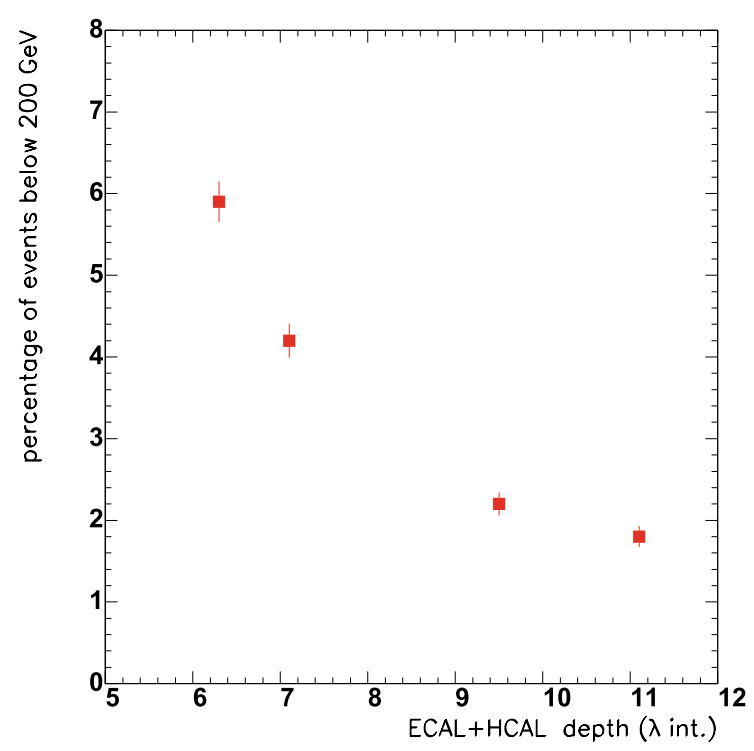

Fig. 3 Fraction of $300 \mathrm{GeV} / \mathrm{c}$ pion data with reconstructed energy less than $200 \mathrm{GeV}$ (approximately $3 \sigma$ below the mean, and corresponding to more than $100 \mathrm{GeV}$ missing energy) versus total absorber depth obtained from 1996 test beam data at $3 \mathrm{~T}$ magnetic field. The total $\lambda_{\text {int }}$ includes the contribution of the electromagnetic calorimeter

\section{The outer hadron calorimeter (HO): constraints and expectation}

The EB plus HB inside the $4 \mathrm{~T}$ cryostat and solenoid is relatively thin. To ensure adequate sampling depth for $\eta<1.4$, the hadron calorimeter is extended outside the cryostat. The $\mathrm{HO}$ will utilise the cryostat and solenoid coil as an additional absorber $\left(1.4 \lambda_{\text {int }} / \sin \theta\right)$ and will also identify and measure the late starting showers.

Outside the cryostat the magnetic field is returned through an iron yoke designed in the form of five $2.536 \mathrm{~m}$ wide (along $z$-axis) rings. The $\mathrm{HO}$ is placed as the first sensitive layer in each of these five rings. The rings are identified by numbers $-2,-1,0,+1$ and +2 . The numbering increases with $z$ and the nominal central $z$ positions of the five rings are respectively $-5.342 \mathrm{~m},-2.686 \mathrm{~m}, 0,+2.686 \mathrm{~m}$ and $+5.342 \mathrm{~m}$. Since the EB plus HB at the central ring (ring 0 ) has the smallest value of $\lambda_{I}$, two layers of HO scintillators were placed on either side of a $19.5 \mathrm{~cm}$ thick piece of iron (the tail catcher iron) at radial distances of $3820 \mathrm{~mm}$ and $4070 \mathrm{~mm}$, respectively. All other rings have a single $\mathrm{HO}$ layer at a radial distance of $4070 \mathrm{~mm}$. The total depth of the calorimeter system is thus extended to a minimum of $10 \lambda_{\text {int }}$ (see Fig. 2) except at the barrel-endcap boundary region.

The HO is constrained by the geometry and construction of the muon system. Figure 1 shows the position of the HO layers in the rings of the muon stations. The segmentation of these detectors closely follows that of the barrel muon system. Each ring has the 12 identical $\phi$-sectors. The 12 sectors are separated by $75 \mathrm{~mm}$ thick stainless steel beams which 
hold successive layers of iron of the return yoke. The space between successive muon rings in the $\eta$ direction and also the space occupied by the stainless steel beams in the $\phi$ direction cause some reduction in the active area of the HO. In addition, the space occupied by the CMS 'chimneys' in the vertical sector of ring -1 and +1 are also not available for the $\mathrm{HO}$ as well. The chimneys are used for the cryogenic transfer lines and power cables of the magnet system. Finally, the mechanical structures needed to position the scintillator trays further constrains $\mathrm{HO}$ along $\phi$.

In the radial direction, space for $\mathrm{HO}$ is limited to only $16 \mathrm{~mm}$ in the iron yoke, as there are aluminium honey comb support structures for the muon detector system. In addition, the HO modules are independently supported from the steel beams located on either side of each $\phi$ sector. The thickness and position of the iron ribs in the yoke structure further constrains the shape and segmentation of the HO.

The sizes and positions of the $\mathrm{HO} 1.0 \mathrm{~cm}$ thick scintillator tiles (BC408) roughly map onto the HB towers of granularity $0.087 \times 0.087$ in $\eta$ and $\phi$. The $\mathrm{HO}$ consists of one (rings \pm 1 and \pm 2 ) or two (ring 0 ) layers of scintillator tiles located in front of the first layer of the barrel muon detector. Scintillation light from the tiles is collected using multi-clad Y11 Kuraray wave-length shifting (WLS) fibres, of diameter $0.94 \mathrm{~mm}$, and transported to the photo detectors located on the structure of the return yoke by splicing a multi-clad $\mathrm{Ku}$ raray clear fibre (also of $0.94 \mathrm{~mm}$ diameter) with the WLS fibre. In order to simplify installation of the HO, the scintillator tiles are packed into a single unit called a tray. Each tray corresponds to one $\phi$ slice $\left(\sim 5^{\circ}\right.$ wide in $\left.\phi\right)$. However, along the $z(\eta)$ direction, a tray covers the entire span of a muon ring. Figure 4 shows a schematic view of a HO tray where one tile is mapped to a tower of the $\mathrm{HB}$ and the optical cable from the tray is connected to the readout box.

The physics impact of HO has been studied [4] using the simulation tool CMSIM developed for the CMS detector. The simulation was carried out for single pions at specific $\eta$ values. The deposited energy in EB plus HB show a deficit in energy measurement starting at $70 \mathrm{GeV} / \mathrm{c}$ at $\eta=0$ (ring 0 ). The results with $\mathrm{HO}$ are more Gaussian in energy distribution indicating that the addition of the $\mathrm{HO}$ properly recovers the energy leakage. Even with $\mathrm{HO}$ in ring 0 there is some small amount of energy leakage. There is evidence of leakage beyond $\mathrm{HO}$ in ring 1 but with reduced intensity. The amount of leakage in ring 2 is found to be negligible.

A critical signature of several new physics signals is the evidence of unobserved particle(s), whether it is supersymmetry, or leptoquark or just plain production of dark matter. CMS detects unobservable particle(s) by measuring missing transverse energy $E_{T}$ and will use it as part of the trigger and data analysis. Any energy that escapes observation will increase the background making data analysis harder. HO has been designed to reduce the unobserved energy background.

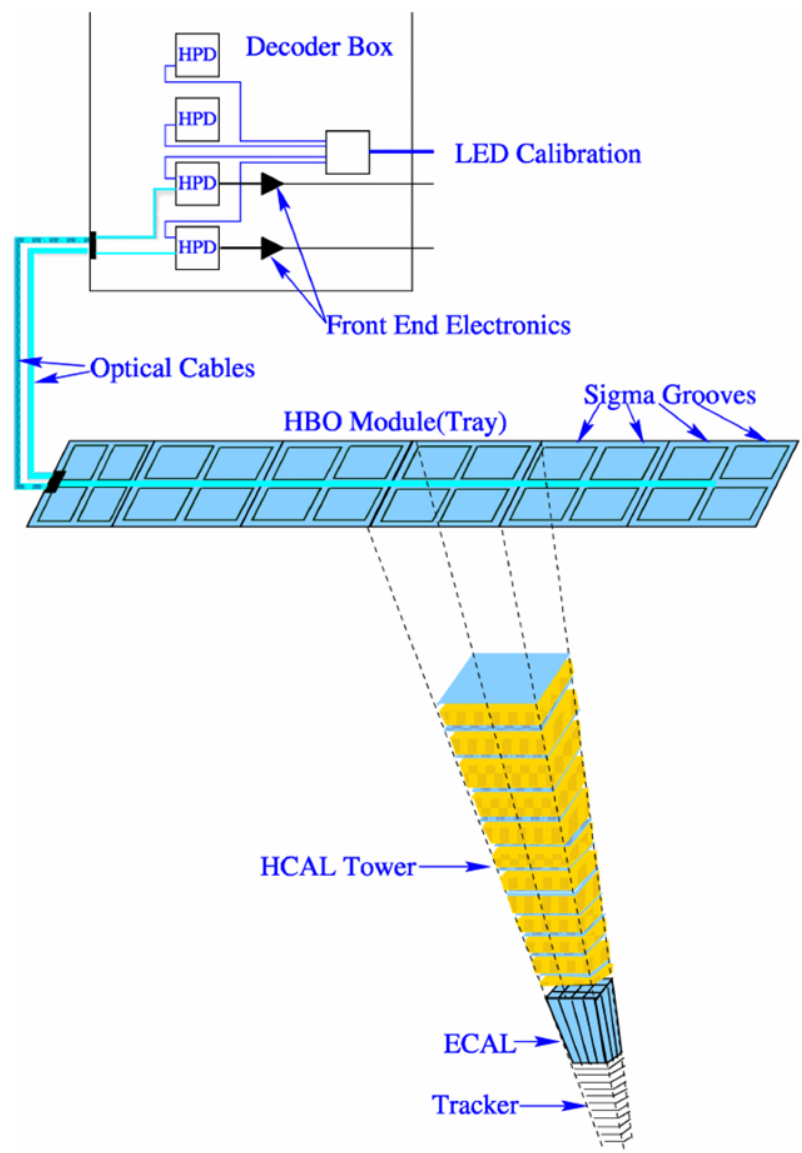

Fig. 4 Schematic view of a HO tray shown with individual tiles and the corresponding grooves for WLS fibres. Each tile is mapped to a tower of HB. Optical fibres from the tray extend to the decoder box which contains the photo-detector and readout electronics

QCD events also have missing $E_{T}$ due to the production of neutrinos. The cross section of QCD events, where at least one particle has $E_{T}$ above $500 \mathrm{GeV}$, is estimated to be several pb. Clearly these events will be affected due to leakage of energy in the hadron calorimeter, and the $\mathrm{HO}$ would help to decrease the background and improve the energy measurement. Figure 5 shows the accepted integrated cross section due to QCD processes for missing $E_{T}$ above a certain value measured with and without HO. It is clear that the inclusion of $\mathrm{HO}$ reduces the background cross section by a factor of 1.5 or more for moderate $E_{T}$ values.

\section{HO module: design and assembly}

\subsection{Mechanical design}

HO [5] is physically divided into 5 rings in $\eta$ conforming to the muon ring structure. The rings are numbered $-2,-1$, $0,+1$ and +2 with increasing $\eta$. Each ring of the $\mathrm{HO}$ is divided into 12 identical $\phi$ sectors (numbered 1 to 12 counting 


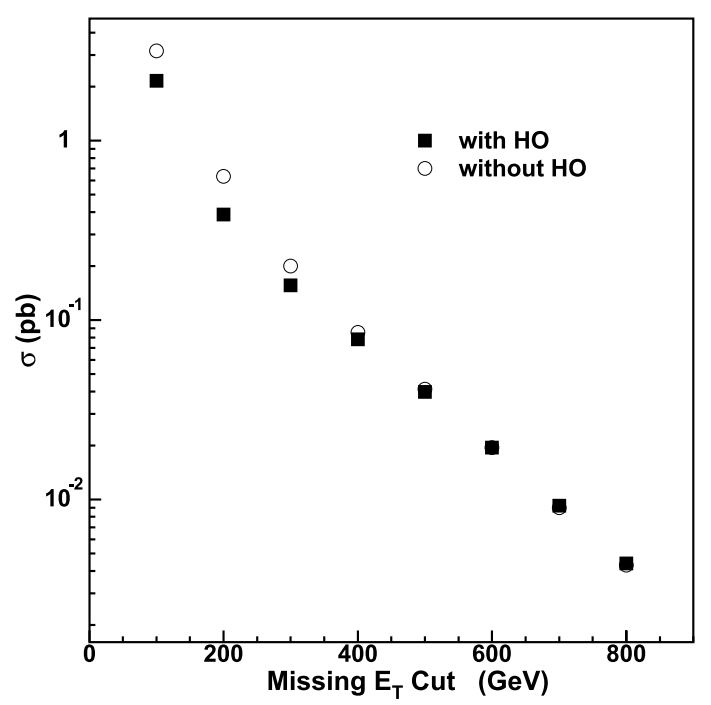

Fig. 5 Accepted integrated cross section above threshold as a function of missing $E_{T}$ when the missing $E_{T}$ is measured with and without the $\mathrm{HO}$

Table 1 Dimension of tiles along $\phi$ for different trays. Each tray corresponds to one $\phi$-slice in a $\phi$ sector

\begin{tabular}{|c|c|c|c|}
\hline \multirow[t]{2}{*}{ Tray \# } & \multicolumn{2}{|l|}{ Ring 0} & \multirow{2}{*}{$\frac{\text { Ring } \pm 1, \pm 2}{\text { Layer } 1}$} \\
\hline & Layer 0 & Layer 1 & \\
\hline Tray 1 & $272.6 \mathrm{~mm}$ & $298.6 \mathrm{~mm}$ & $315.6 \mathrm{~mm}$ \\
\hline Tray 2 & $341.6 \mathrm{~mm}$ & $362.6 \mathrm{~mm}$ & $364.6 \mathrm{~mm}$ \\
\hline Tray 3 & $330.6 \mathrm{~mm}$ & $350.6 \mathrm{~mm}$ & $352.6 \mathrm{~mm}$ \\
\hline Tray 4 & $325.6 \mathrm{~mm}$ & $345.6 \mathrm{~mm}$ & $347.6 \mathrm{~mm}$ \\
\hline Tray 5 & $325.6 \mathrm{~mm}$ & $345.6 \mathrm{~mm}$ & $347.6 \mathrm{~mm}$ \\
\hline Tray 6 & $266.6 \mathrm{~mm}$ & $290.6 \mathrm{~mm}$ & $404.6 \mathrm{~mm}$ \\
\hline
\end{tabular}

clockwise starting from 9 o'clock position) and each sector has 6 slices (numbered 1 to 6 counting clockwise) in $\phi$. The $\phi$ slices of a layer are identical in all sectors. The widths of the slices along $\phi$ are given in Table 1. In each $\phi$ slice, there is a further division along $\eta$. The smallest scintillator unit is called a tile. The scintillator tiles in each $\phi$ sector belong to a plane. Perpendicular distance of this plane from the $z$-axis is $3.82 \mathrm{~m}$ for layer 0 and $4.07 \mathrm{~m}$ for layer 1 . The tiles in each $\phi$ slice of a ring are mechanically held together in the form of a tray (details in Sect. 3.2).

Both layers of ring 0 have $8 \eta$-divisions (i.e. 8 tiles in a tray): $-4,-3,-2,-1,+1,+2,+3,+4$. Ring 1 has 6 divisions: $5, \ldots, 10$ and ring 2 has 5 divisions: $11, \ldots, 15$. Ring -1 and ring -2 have the same number of divisions as rings 1 and 2 but with negative indices. The $\eta$-dimensions of any tile with negative tower number is the same as the one with positive number. Tile dimensions along $\eta$ for the towers are shown in Table 2.

Length of a full tray is $2510 \mathrm{~mm}$ whereas the shorter trays, the sizes of which are constrained because of the
Table 2 HO tile dimensions along $\eta$ for different rings and layers. The tile sizes, which are constrained by muon ring boundaries, are mentioned in parenthesis

\begin{tabular}{|c|c|c|c|c|c|}
\hline Tower & $\eta_{\max }$ & $\begin{array}{l}\text { Length } \\
(\mathrm{mm})\end{array}$ & Tower & $\eta_{\max }$ & $\begin{array}{l}\text { Length } \\
(\mathrm{mm})\end{array}$ \\
\hline \multicolumn{2}{|c|}{ Ring 0 Layer 0} & & \multicolumn{2}{|c|}{ Ring 0 Layer 1} & \\
\hline 1 & 0.087 & 331.5 & 1 & 0.087 & 351.2 \\
\hline 2 & 0.174 & 334.0 & 2 & 0.174 & 353.8 \\
\hline 3 & 0.262 & 339.0 & 3 & 0.262 & 359.2 \\
\hline 4 & 0.326 & $(248.8)$ & 4 & 0.307 & $(189.1)$ \\
\hline \multicolumn{2}{|c|}{ Ring 1 Layer 1} & & \multicolumn{2}{|c|}{ Ring 2 Layer 1} & \\
\hline 5 & 0.436 & 391.5 & 11 & 0.960 & 420.1 \\
\hline 6 & 0.524 & 394.2 & 12 & 1.047 & 545.1 \\
\hline 7 & 0.611 & 411.0 & 13 & 1.135 & 583.3 \\
\hline 8 & 0.698 & 430.9 & 14 & 1.222 & 626.0 \\
\hline 9 & 0.785 & 454.0 & 15 & 1.262 & (333.5) \\
\hline 10 & 0.861 & (426.0) & & & \\
\hline
\end{tabular}

chimney (trays 4 and 5 in sector 4 of ring +1 and trays 3 , 4,5 and 6 in sector 3 of ring -1 ), are $2119 \mathrm{~mm}$ long. The shorter trays are constructed without the tile corresponding to tower number \pm 5 . Because of the constraints imposed by the gap between the two rings, a part of tower $\# \pm 4$, which falls in ring \pm 1 (tower \pm 4 is restricted only to ring 0 ) is merged with tower $\# \pm 5$.

There is a maximum of $40 \mathrm{~mm}$ of clearance for placing the HO trays together with the mechanical structures. Each tray with all its packing is expected to have a thickness of $15.44 \mathrm{~mm}$. This necessitated a space of $17.0_{-0.0}^{+0.2}$ $\mathrm{mm}$ for inserting the trays. The total weight of a $\phi$ sector (six trays) is about $30.0 \mathrm{~kg}$. To support this weight, a pair of aluminium honeycomb panels $(10 \mathrm{~mm}$ thick on the bottom and $6 \mathrm{~mm}$ thick at the top) are clamped between three aluminium $\mathrm{C}$-channels on the two sides and one at the end while the other end is kept open for inserting the trays. A set of twelve $20 \mathrm{~mm} \times 20 \mathrm{~mm}$ aluminium C-channels are glued on the inner side at a fixed separation for inserting the trays. The separation is properly adjusted for the appropriate tray width. All the panels are $2530_{-0}^{+5} \mathrm{~mm}$ long. The widths of the three types of panels (layer 0 and 1 of ring 0 and layer 1 of rings $\pm 1, \pm 2$ ) are respectively $1920_{-0}^{+2} \mathrm{~mm}, 2050_{-0}^{+2}$ $\mathrm{mm}$ and $2190_{-0}^{+2} \mathrm{~mm}$. Panels for rings \pm 1 and \pm 2 also carry cooling tubes on one side. These serve as thermal screens to prevent heat percolation to the RPC/muon chamber assembly. The cooling tubes are made of copper and have inner and outer diameter of $6 \mathrm{~mm}$ and $8 \mathrm{~mm}$. These are glued to the $6 \mathrm{~mm}$ thick honeycomb surface using araldite and fixed with copper collars. For rings \pm 1 , a special panel is built to accommodate the short trays due to the chimneys. 

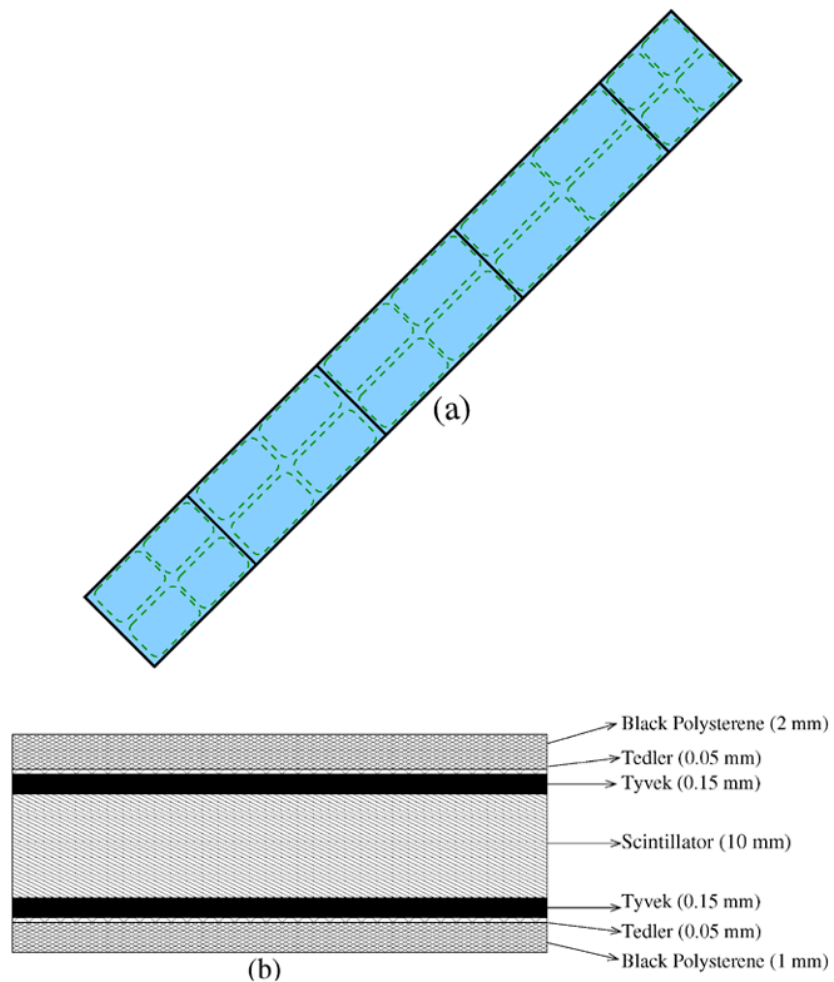

Fig. 6 (a) Layout of scintillator tiles in a typical tray of ring 2. (b) Cross section of a $\mathrm{HO}$ tray showing the different components

\subsection{Trays}

All tiles in each $\phi$ slice of a sector are grouped together in the form of a tray. Figure 6(a) shows a schematic view of a $\mathrm{HO}$ tray. Each tray contains 5 tiles in rings $\pm 2 ; 6$ tiles in rings \pm 1 and 8 tiles in ring 0 . The edges of the tiles are painted with Bicron reflecting white paint for better light collection as well as isolating the individual tiles of a tray. Further isolation of tiles is achieved by inserting a piece of black tedlar strip in between the adjacent tiles. The tiles in a tray are covered with a single big piece of white, reflective tyvek paper. Then they are covered with black tedlar paper to prevent light leak. This package is placed between two black plastic plates for mechanical stability and ease of handling. The top plastic cover is $2 \mathrm{~mm}$ thick and the bottom one is $1 \mathrm{~mm}$ thick. Figure 6(b) shows a cross section of a tray to illustrate the different components.

The $2 \mathrm{~mm}$ plastic sheet on the top has $1.6 \mathrm{~mm}$ deep channels grooved on it (on the outer side) to route the fibres from individual tiles to an optical connector placed in a groove at the edge of the tray. A $1.5 \mathrm{~mm}$ wide straight groove runs along the edge of the top cover to accommodate a stainless steel tube. This is used for the passage of a radioactive source for calibrating the modules. Each connector has two holes and these are fixed to the scintillator-plastic assembly through matching holes in them. Each $\phi$ sector in each ring

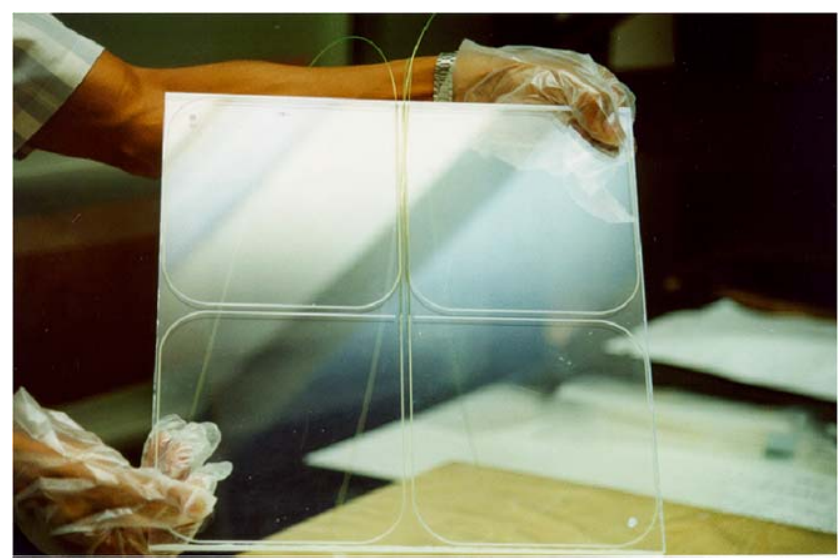

Fig. 7 View of a typical HO tile with WLS fibres inserted in the 4 $\sigma$-grooves

has 6 trays. There are 360 trays for layer 1 and 72 trays for layer 0 .

\subsection{Tiles}

Scintillator tiles are made from Bicron BC408 scintillator plates of thickness $10_{-1}^{+0} \mathrm{~mm}$. Figure 7 shows a typical HO scintillator tile. The WLS fibres are held inside the tile in grooves with a key hole cross section. Each groove has a circular part (of diameter $1.35 \mathrm{~mm}$ ) inside the scintillator and a neck of $0.86 \mathrm{~mm}$ width. The grooves are $2.05 \mathrm{~mm}$ deep. Each tile has 4 identical $\sigma$-shaped grooves, one groove in each quadrant of the tile. The grooves closely follow the quadrant boundary. The corners of the grooves are rounded to prevent damage to the fibre at the bend and to ease fibre insertion. The groove design is slightly different for the tile where the optical connector is placed at the end of the tray. Since the tiles are large, 4 grooves ensure good light collection and reduced attenuation of light.

\subsection{Pigtails}

The light collected by the WLS fibres inserted in the tiles is transported to photo-detectors. Captive ends of the WLS fibres, which reside inside the groove, are polished, aluminised and protected using a thin polymer coating. The other end of the WLS fibre comes out of the tile through a slot made on the $2 \mathrm{~mm}$ thick black plastic cover sheet. To minimise the loss of light in transportation, the WLS fibre (attenuation length of $\sim 1.8 \mathrm{~m}$ ) is spliced to a clear fibre (attenuation length of $\sim 7.0 \mathrm{~m}$ ). The clear fibres from each tile follow the guiding grooves on the top plastic to the optical connector at the end. Each tray has two optical connectors mounted on one end of the tray.

The pigtails are tested with the help of a specially built scanner. A UV lamp moves over the pigtail in steps under a computer controlled mechanical stage. At specific intervals, 


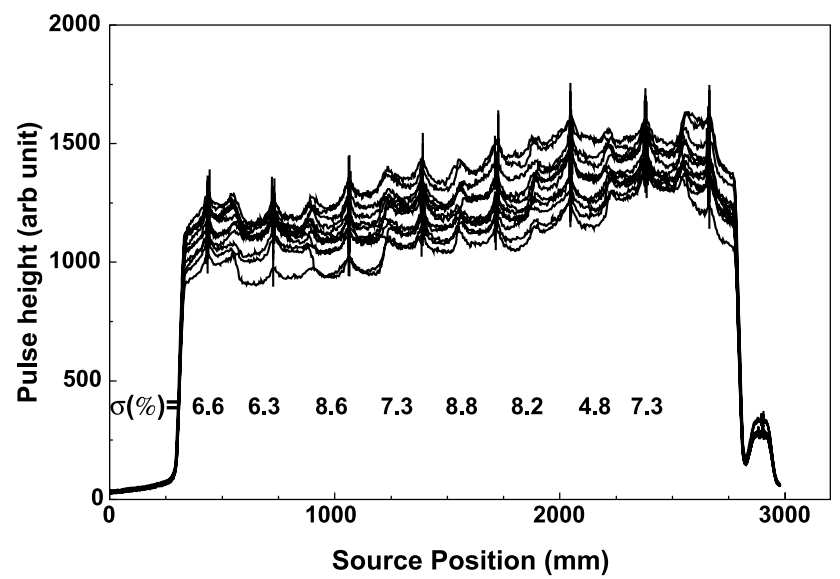

Fig. 8 Scan of a set of 12 fully assembled scintillator trays (of the 12 sectors) belonging to ring 0 layer 0 tray position 3 using the XY scanner. The lines on the figures refer to the peak positions corresponding to the centre of the tiles. The tiny peak on the right side is due to a calibration tile used in each set of measurements

the UV lamp shutter opens, the fibres absorb UV photons and the re-emitted light in each fibre is transmitted to the end to a set of photo diodes. Signals from the photo diodes are then digitised and analysed to study the combined transmission loss due to the spliced junctions, connector interface and attenuation. The mean transmission efficiency and its spread $\sigma$ over the entire production was found to be about 95\% and $3 \%$ respectively.

\subsection{Quality assurance}

Each tray is subjected to quality assurance tests using a moving radioactive source and cosmic ray muons. The radioactive source moves in $\mathrm{X}$ and $\mathrm{Y}$ direction (XY scanner) under computer control and the output is read by a data acquiring system. There is an additional set of measurements using radioactive source on a tip of a moving wire through the stainless steel tube fixed to each assembled tray.

$\mathrm{XY}$ scanner.

Figure 8 shows the ADC output at several $x$ and $y$ points over the surfaces of twelve trays belonging to layer 0 of ring 0 . The collimated source excites all tiles in a similar way. One clearly sees that signal size for tiles close to the connector is largest and it slowly decreases for tiles with larger fibre length. The fluctuations among the trays are at a level much below $10 \%$ which is used as the acceptance criterion for the trays.

Wire source.

The tiles are also inter-calibrated using radioactive source technique [6] using a $\mathrm{Co}^{60}$ source with a strength of $5 \mathrm{mCi}$. Signals are sampled at the rate of $40 \mathrm{MHz}$ and data are taken in histogramming mode. The mean of such a histogram represents the response of tile at the given reel position. While analysing the wire source data, the reel position is used to
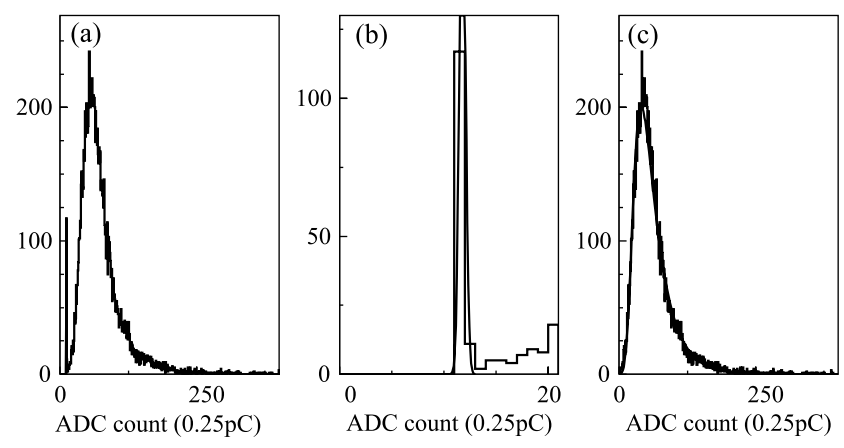

Fig. 9 (a) Raw ADC spectrum for tile \# 8 for one of the trays of layer 0, ring 0. (b) A fit to the pedestal part to a Gaussian distribution. (c) Pedestal subtracted distribution fit to a Gaussian convoluted by Landau distribution

find out the correspondence between tile number and readout channel. This method is also used during the beam test operation.

Cosmic ray muons.

The HO trays are expected to supply at least 10 photo electrons for a minimum ionising particle (MIP) if a photomultiplier tubes (PMT) were used in the read out. The completed trays have been tested for their responses to MIPs using cosmic rays in a special stand designed for this purpose. Signal from the tiles are grouped into two bunches (alternate tiles) and the two bunches are read by two calibrated green extended PMT's. The electronic signal is sent to a chargesensitive ADC. These PMT's are calibrated using a precisely controlled LED source to get the calibration for ADC count per photo electron.

Data are monitored on-line, and there is a calibration tile equipped with fibre readout which is placed separately and is always read out to monitor PMT gain. The pulse height spectrum is analysed offline to determine the number of primary photoelectrons produced. Figure 9(a) shows a typical ADC spectrum. One sees a very clear peak due to the passage of cosmic muons well separated from the pedestal peak. The pedestal subtracted pulse height (see Fig. 9(c)) is fit to a Landau distribution and the position of the mean value of the spectrum together with the calibration factor provides the number of photoelectrons yielded in a given tile.

Figure 10 shows a plot of average number of photoelectrons obtained using cosmic muons as a function of the tile number in a set of 12 trays belonging to ring 0 layer 0 tray. The number of photo electrons is always above 10 . The number of photoelectrons are as high as 20 for some of the tiles close to the connector. The RMS spread in the number of photo-electrons is less than $10 \%$ which is the acceptance criterion for the trays.

Correlation between all the tests (with cosmic muons, source tube and XY scan) are shown in Fig. 11. The same set of readout system is used for cosmic and wire source test, whereas a different PMT, optical connectors are used 
in the XY-scanner. Statistical error on these measured variables are less than $1 \%$. HO towers will be calibrated in situ using muons from Cosmic Rays or from collision events. The strong correlation among the three set of measurements validates the use of muons in calibrating the system.

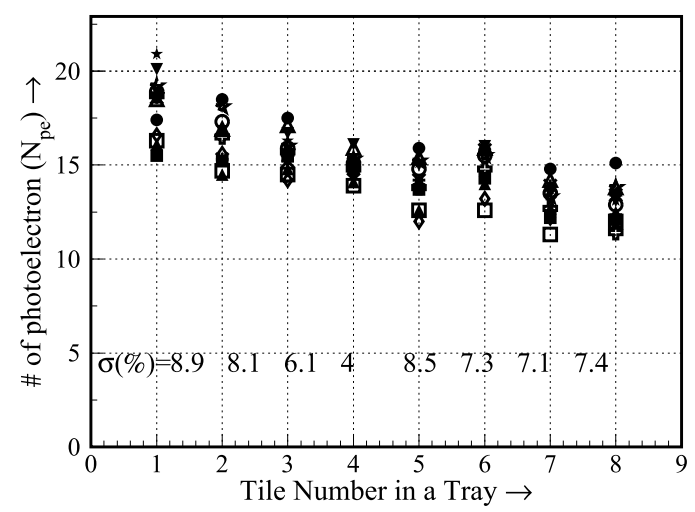

Fig. 10 Number of photo electrons for the tiles in fully assembled scintillator trays of layer 0 , ring 0 . Tile number is counted from the tray end with optical connectors. There are 12 measurements for a given tile number coming from 12 trays and the measurements have a spread between (4.0-8.9)\% depending on the tile position
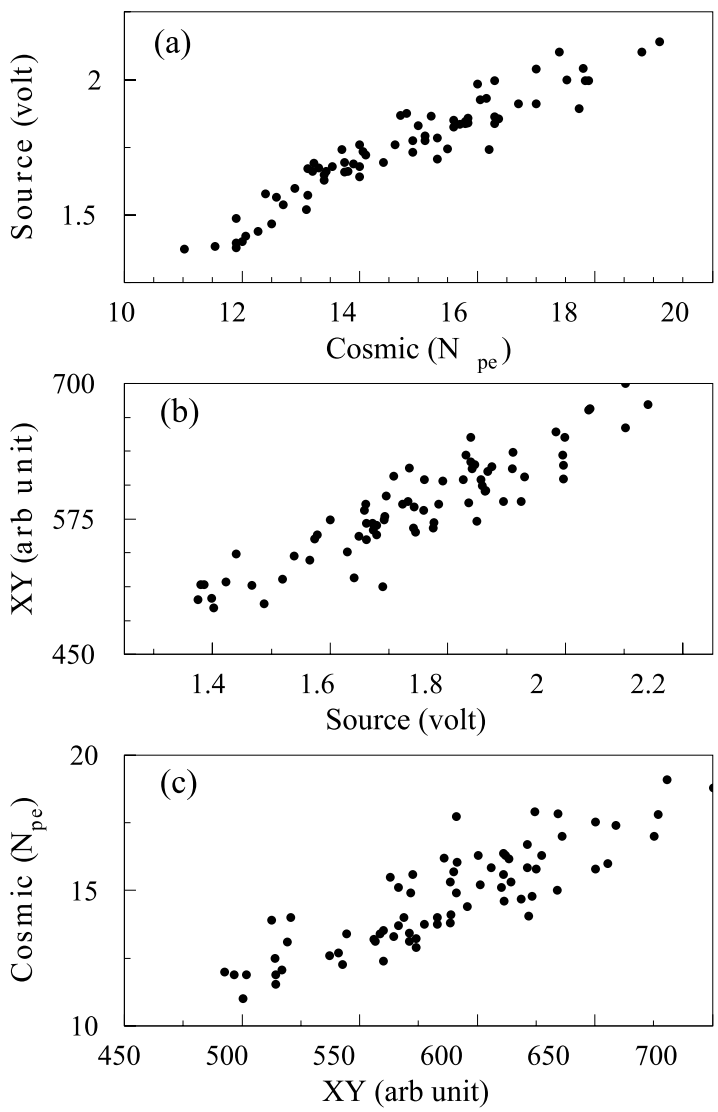

Fig. 11 Correlations between number of photo-electron in cosmic test and signal peaks in the wire source and XY source test

\section{Test beam studies}

Prototypes of $\mathrm{HO}$ trays were put together with 2 wedges of $\mathrm{HB}$ and a prototype of one sector of endcap hadron calorimeter module (HE) in the test beam facility at CERN. These prototype trays were exposed to a variety of charged beams of hadrons, electrons and muons over a wide energy range. These tests have been carried out over a number of years.

The signals from these detectors are read out using Hybrid Photo Diodes (HPD) which converts the light into electrical charges. These are then measured and encoded into a non-linear digital scale by the charge integrator IC (QIE). The QIE uses LHC clock to divide time into regular bins of $25 \mathrm{~ns}$ and measures the accumulated charge in each time bin. Internally, the QIE uses capacitors to accumulate the charge. The QIE's use non linear scale so that the QIE step size is a approximate constant fraction of the QIE value.

Arrival time of signals from a scintillator tile to the readout element has a significant dependence on its location in $\eta$. Energy is measured by summing over a number of time steps and this $\eta$ dependent time of arrival makes the energy measurement rather complex. This effect has been corrected by introducing an appropriate $\eta$ dependent delay for each of the towers (see Fig. 12).

The HO trays were calibrated using the radioactive wiresource test as well as by exposing them to a beam of muons. Figure 13 shows a plot of ADC counts from ring 0 , layer 0 tiles as the radioactive source passes along them. Pedestal is determined by fitting a straight line with zero slope to the part of the signal when radioactive source is far away from the tile under consideration. It is evident from the figure that there is a leakage of the signal to the neighbouring tiles when the source is at the edge of the tile. So the pedestal subtracted signals from the neighbouring tiles are added with proper weight to get the total signal. The signal from a tile and that

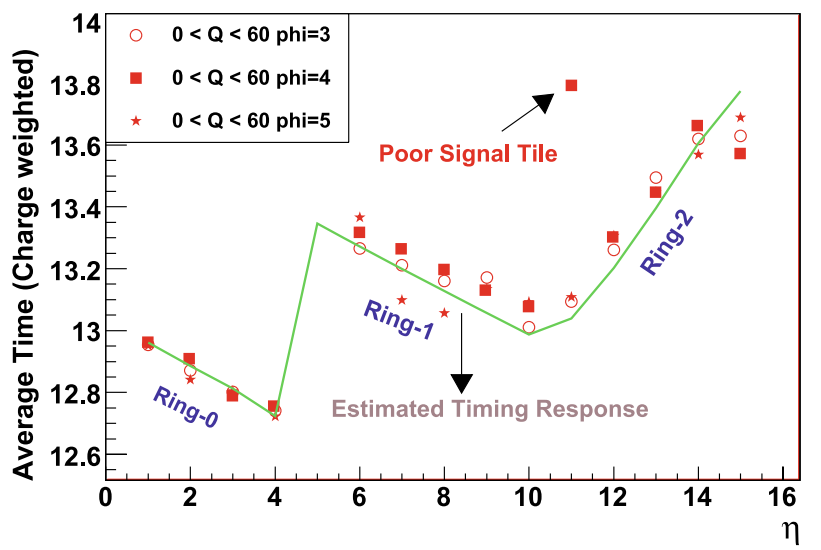

Fig. 12 Charge weighted average time slice as a function of $\eta$ index of the tiles in $\mathrm{HO}$ before the time delay correction. The solid line is the estimated time delay as a function of rapidity. The vertical axis is in units of $25 \mathrm{~ns}$ 


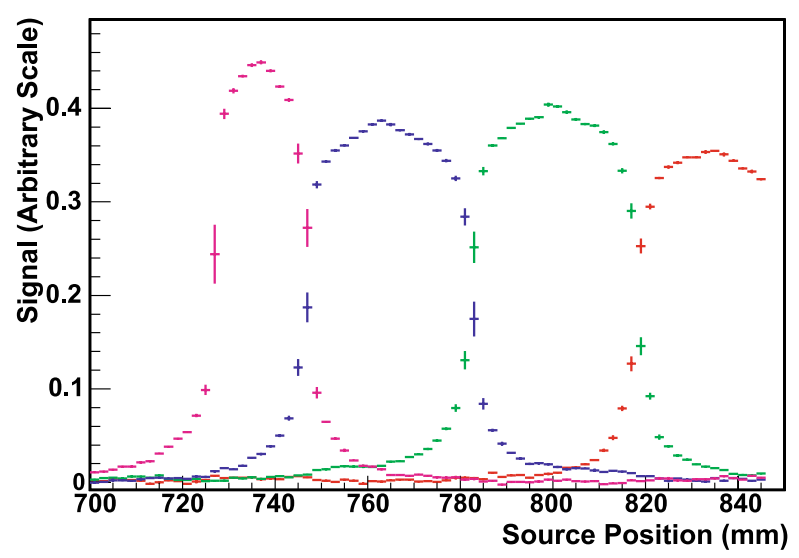

Fig. 13 ADC counts from ring 0, layer 0 tiles as the radioactive source passes along them

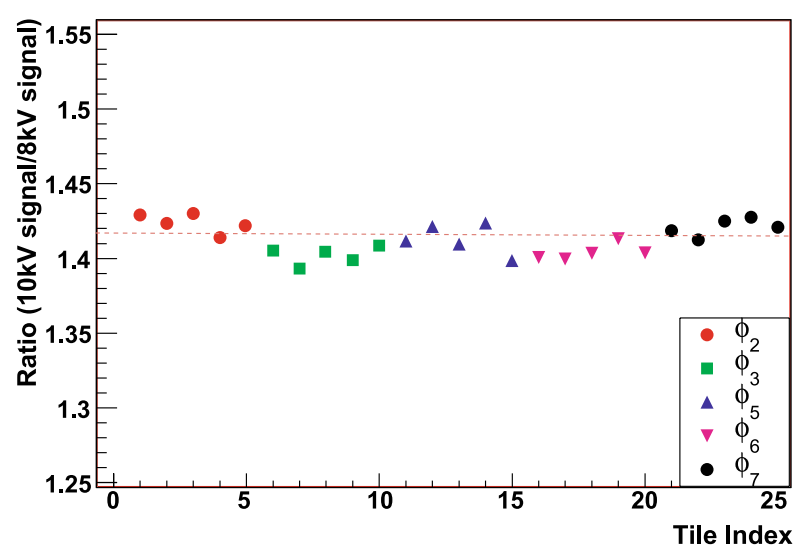

Fig. 14 Ratio of calibration constant for ring $1 \mathrm{HO}$ tiles in the test beam set up with the HPD's being operated at $10 \mathrm{kV}$ and at $8 \mathrm{kV}$

from its two adjacent ones are then fitted using an iterative procedure to extract the calibration constants. The HPD for HO readout was operated at voltages $8 \mathrm{kV}$ and $10 \mathrm{kV}$ and separate datasets were collected for the two settings. Calibration constants obtained with these two sets of data show the expected consistency (see Fig. 14) being different by a factor of 1.415 .

For the muon runs, the HPD was operating at $10 \mathrm{kV}$. Muons of energy $150 \mathrm{GeV}$ were pointed at the centre of each $\mathrm{HO}$ tile and the ADC signal from the tile was used to determine the muon calibration constant. To minimise noise, the sum of 4 time slices with the maximum amount of energy deposit is taken as the signal. The sum of four time slices away from the signal region is taken as the pedestal. The pedestal and signal peaks are not well separated in all the tiles (particularly in ring 1). The pedestal value is taken as the fitted mean obtained by fitting the pedestal distribution with a Gaussian curve. The peak region of the pedestal subtracted muon signal is then fitted with a Gaussian convoluted Landau distribution and the most probable value of this distribution from the fit is taken as the muon calibra-

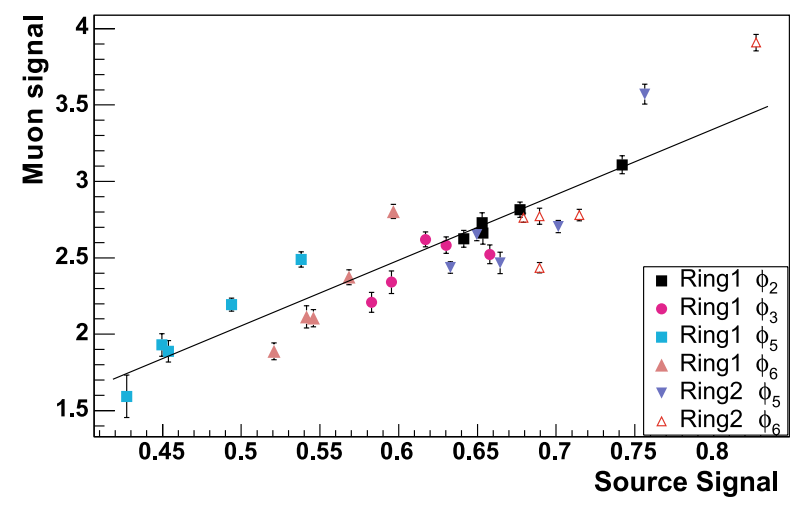

Fig. 15 Calibration constant from muon signal plotted against the corresponding value from the wire source analysis

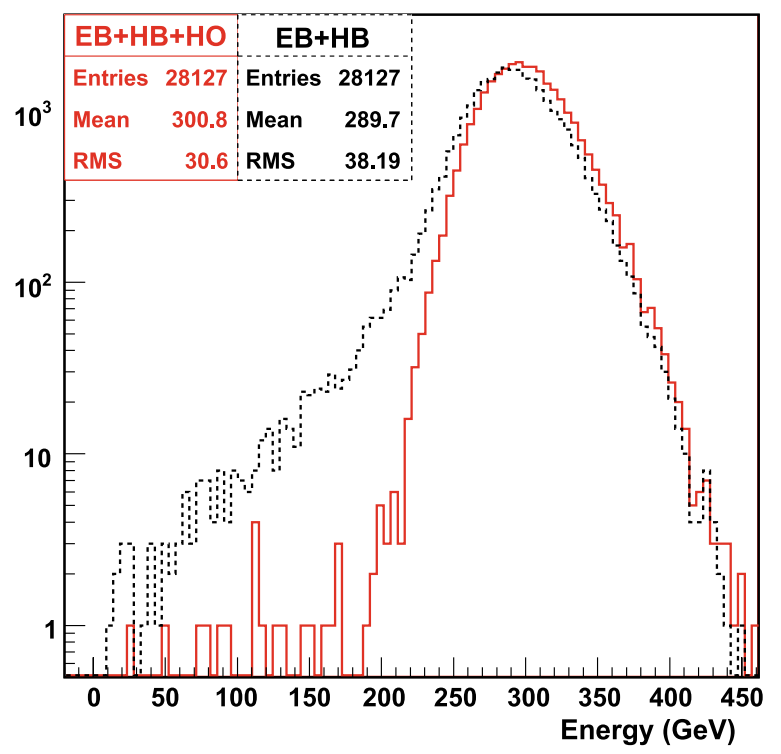

Fig. 16 Energy distribution for a $300 \mathrm{GeV}$ pion beam measured with $\mathrm{EB}+\mathrm{HB}$ and with $\mathrm{EB}+\mathrm{HB}+\mathrm{HO}$

tion constant for the tile. Figure 15 shows correlation plot of calibration constants obtained through the two sets of measurements.

Figure 16 shows energy distributions for a $300 \mathrm{GeV}$ pion beam with only $\mathrm{EB}+\mathrm{HB}$ in the beam and with $\mathrm{EB}+\mathrm{HB}+\mathrm{HO}$ in the beam. The measured distributions for hadron beams of energy above $100 \mathrm{GeV}$ are more symmetric and have smaller widths after adding HO to the energy calculation. This confirms the expectations from simulation studies shown in Sect. 2.

Weight factor for $\mathrm{HO}$ has been found to be $372 \mathrm{MeV} / \mathrm{fC}$ for beam being shot at $\eta=0.22$ (Ring 0) and $407 \mathrm{MeV} / \mathrm{fC}$ at $\eta=0.56$ (Ring 1). Energy resolution for $\pi^{-}$beam has been measured as a function of beam energy at those two configurations and are shown in Fig. 17. The two sets of measurements in each plot refer to measurements without and with HO. As can be seen in the figure, the energy reso- 

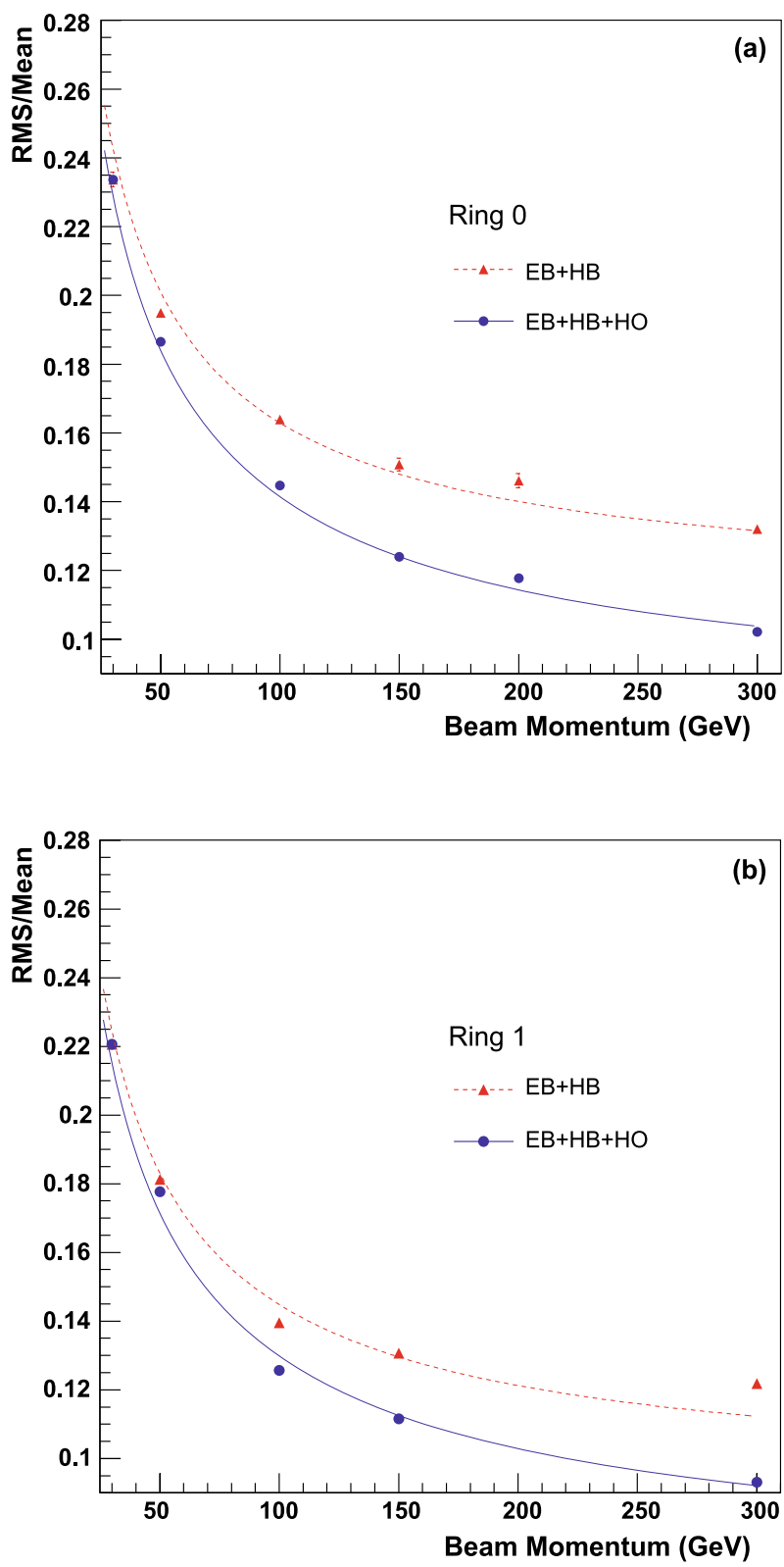

Fig. 17 Energy resolution for pions as a function of beam energy measured with $\mathrm{EB}+\mathrm{HB}$ and with $\mathrm{EB}+\mathrm{B}+\mathrm{HO}$ for the beam being shot at (a) $\eta=0.22$ and (b) $\eta=0.56$

lution improves significantly with the inclusion of HO. Fits to the resolution distribution indicate that the constant term improves from $11.2 \%$ to $7.8 \%$ for Ring 0 and from $9.2 \%$ to $6.6 \%$ for Ring 1 with the inclusion of $\mathrm{HO}$.

The signal size due to penetrating beam is compared with the noise level in the HO tiles from the 2002 test beam studies (see Fig. 18). This study has indicated that HO will be able to provide signals for minimum ionising particles with efficiency better than $90 \%$ keeping the noise level below 20\%. CMS uses signal in the Resistive Plate Chambers (RPC) to trigger for muons in the barrel as well as in the endcap region. In the barrel (RPC trigger towers 1-6) and in the
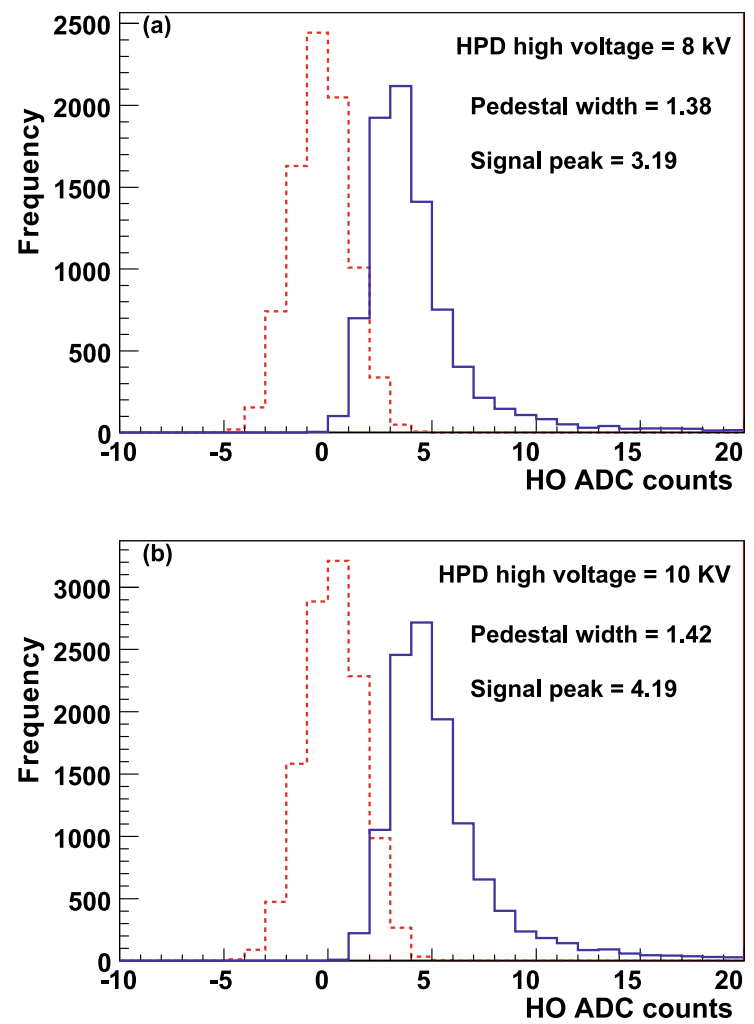

Fig. 18 Pedestal peak and muon signal for a ring 2 tile operated with a voltage of (a) $8 \mathrm{kV}$, (b) $10 \mathrm{kV}$ on the HO HPD

overlap region (RPC trigger towers 7-9) for the RPC's, there is a considerable coverage by HO. Even with $95 \%$ chamber efficiency of the RPC's, muon trigger efficiency is rather poor for RPC trigger towers 6-9 (as low as 72\%) if only RPC's are used in coincidence. This study suggested that $\mathrm{HO}$ could be a useful component in muon trigger together with the RPC at these solid angle [7].

As it has been observed for data collected with the radioactive source, size of the signal collected from HO tiles is considerably bigger when the HPD is operated at $10 \mathrm{kV}$ as compared to $8 \mathrm{kV}$. This is also demonstrated in the HO signals obtained from the 2002 test beam studies with $225 \mathrm{GeV} / \mathrm{c}$ muon beam, as shown in Fig. 18. Use of HO will make the trigger efficiency better than $90 \%$ over most of the solid angle. Figure 19 shows a plot of efficiency of muon detection versus purity of the signal obtained from the 2004 test beam studies.

\section{Summary}

Constrained to lie outside of the inner CMS detector layers (pixels, tracker and electromagnetic calorimeter) and inside the 4 Tesla magnet, the hadron barrel calorimeter was found to be too thin to effectively absorb high energy hadrons specially near the $\eta=0$ region. This would lead to degraded 


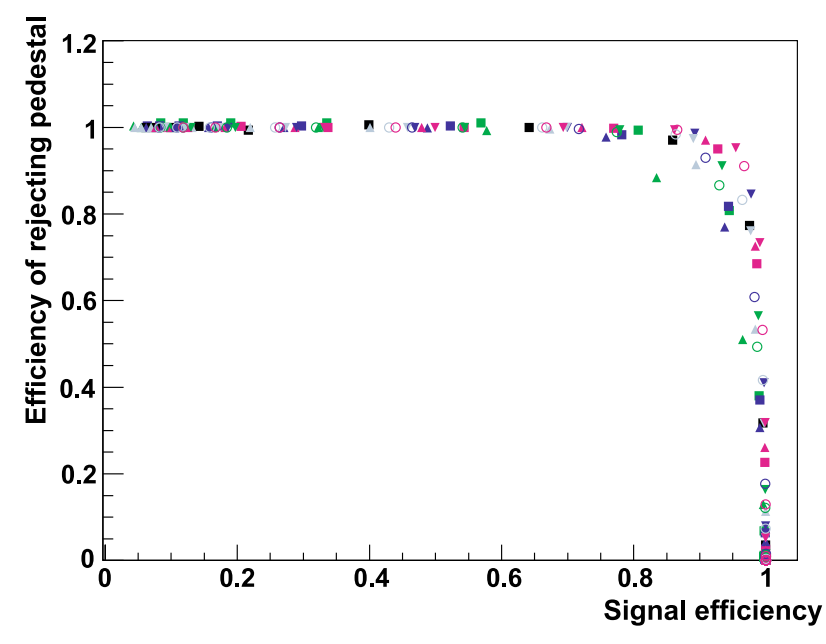

Fig. 19 Efficiency of rejecting pedestal vs. efficiency of muon detection in the $\mathrm{HO}$ detector. Different markers refer to different read out towers in ring 0

sensitivity for establishing new physics signals whose main signature is missing $E_{T}$. The addition of sensitive detector layers just outside the magnet (inside the first barrel muon station) was simulated and shown to ensure much smaller energy leakage and hence improvement in the energy resolution.

Sensitive detector layers of $1 \mathrm{~cm}$ thick plastic scintillator with embedded WLS fibre readout (similar technology as the CMS barrel and endcap hadron calorimeters) were selected as the appropriate detectors.

Acknowledgements The results presented in this paper are partially based on the doctoral thesis of Seema Sharma [8]. This project was carried out with financial support from CERN, Department of Atomic Energy and Department of Science and Technology of India, U.S. Department of Energy, U.S. National Science Foundation, RMKI-KFKI (Hungary, OTKA grant T 016823), Federal Agency for Science and Innovations of the Ministry for Education and Science of the Russian Federation, Russian Academy of Sciences, Scientific and Technical Research Council of Turkey (TUBITAK), Turkish Atomic Energy Agency (TAEK), Bogazici University Research Grant (Grant no: 04B301).

\section{References}

1. CMS Technical Proposal, CERN/LHCC 94-38, LHCC/P1, 15 December 1994

2. CMS Hadron Calorimeter Technical Design Report, CERN/LHCC 97-31, CMS TDR 2, 20 June 1997

3. V.V. Abramov et al., CMS-HCAL Collaboration, Nucl. Instrum. Methods A 457, 75 (2001)

4. S. Banerjee, S. Banerjee, CMS Note 1999/063

5. Outer Hadron Calorimeter Engineering Design Review, TIFR/CMS-99-01

6. E. Hazen et al., Nucl. Instrum. Methods A 511, 311 (2003)

7. C. Albajar et al., CMS Note 2003/009

8. S. Sharma, Ph.D. thesis, Tata Institute of Fundamental Research, Mumbai, 2008 (unpublished) 